\title{
Computed Tomography of the Esophagus in Scleroderma and Lung Disease
}

\author{
Daisuke Takekoshi, ${ }^{1}$ Shiva Arami, ${ }^{2}$ Todd J. Sheppard, ${ }^{1}$ Patricia Cole-Saffold, ${ }^{1}$ \\ Jon C. Michel, ${ }^{1}$ George T. Kondos ${ }^{3}$ and Dean E. Schraufnagel ${ }^{1}$ \\ ${ }^{1}$ Division of Pulmonary, Critical Care, Sleep and Allergy, Department of Medicine, University of Illinois at \\ Chicago, Chicago, IL, USA \\ ${ }^{2}$ Division of Rheumatology, Department of Medicine, University of Illinois at Chicago, Chicago, IL, USA \\ ${ }^{3}$ Division of Cardiology, Department of Medicine, University of Illinois at Chicago, Chicago, IL, USA
}

\begin{abstract}
Systemic sclerosis, or scleroderma, is a collagen vascular disease characterized by hardening of the skin and involvement of internal organs, most commonly the esophagus. The most frequent cause of death in these patients is lung disease. Esophageal dysfunction has been implicated in the pathogenesis of interstitial lung disease. We previously developed a standard for the esophageal diameter on chest computed tomography (CT) and hypothesized that patients with esophageal dilation would be more likely to have interstitial lung disease than those without. In this study, we test this in 121 systemic sclerosis patients with interstitial lung disease and 48 of those without interstitial lung disease. For controls, we evaluated 121 patients followed at a general pulmonary clinic and the previously studied normal healthy standards. This study demonstrated that esophageal dilation is common in systemic sclerosis patients (66.3\% for the maximal esophageal diameter more than or equal to $15 \mathrm{~mm}$ ), that systemic sclerosis patients with interstitial lung disease have more dilated esophagi than those without interstitial lung disease (median $19.4 \mathrm{~mm}$ vs. $14.1 \mathrm{~mm}$ ), and that esophageal parameters are negatively correlated with pulmonary function. We also found that patients from general pulmonary clinic were more likely to have dilated esophagi than normal controls (median $12.1 \mathrm{~mm}$ vs. $9.7 \mathrm{~mm}$ ). The CT measurement of esophageal diameter may be a useful marker of patients at risk for developing lung disease.
\end{abstract}

Keywords: computed tomography; esophageal dilation; interstitial lung disease; reflux disease; systemic sclerosis Tohoku J. Exp. Med., 2015 December, 237 (4), 345-352. (C) 2015 Tohoku University Medical Press

\section{Introduction}

Systemic sclerosis, or scleroderma, is a collagen vascular disease characterized by thickening and hardening of the skin as well as various internal organs. The most common organ involved is the esophagus. Systemic sclerosis is more prevalent in Blacks than Whites, somewhat lower in Asians, and unknown for Hispanics (Reveille 2003).

We previously reported the normal esophageal measurements on chest computed tomography (CT) scan based on a normal general population (Schraufnagel et al. 2008). The prevalence of esophageal dilation using our upper limit of normal in patients with systemic sclerosis or other pulmonary diseases is unknown.

Esophageal dilation is commonly seen on chest $\mathrm{CT}$ in patients with systemic sclerosis (Bhalla et al. 1993). While several studies reported the prevalence of esophageal dilation on chest CT scans for systemic sclerosis patients, these studies used empirical cutoff values to define esophageal dilation without regard to normal standards (Bhalla et al. 1993; Pitrez et al. 2006; Vonk et al. 2008; Patiwetwitoon et al. 2012).

Lung involvement is also common in systemic sclerosis and is currently the leading cause of death in this disease (Steen and Medsger 2007; Christmann et al. 2010). It has been suggested that esophageal dysfunction and aspiration may play a role in pathogenesis of interstitial lung disease in systemic sclerosis and other lung diseases (el-Serag and Sonnenberg 1997; Tobin et al. 1998; Marie et al. 2001; Savarino et al. 2009; Christmann et al. 2010). Esophageal dilation on CT scan correlates well with esophageal dysfunction (Pitrez et al. 2006; Vonk et al. 2008). The CT image of esophagus, if correlated with function, has several practical advantages over conventional methods to evaluate esophageal dysfunction: availability, noninvasiveness, easy repeatability, and little need of patient cooperation. However, previous studies demonstrated conflicting results in the correlation between esophageal dilation on $\mathrm{CT}$ and interstitial

Received June 22, 2015; revised and accepted November 6, 2015. Published online December 5, 2015; doi: 10.1620/tjem.237.345.

Correspondence: Dean E. Schraufnagel, M.D., Division of Pulmonary, Critical Care, Sleep and Allergy, Department of Medicine,

University of Illinois at Chicago, M/C 719, 840 South Wood St., Chicago, IL 60612-7323, USA.

e-mail: schrauf@uic.edu 
lung disease in systemic sclerosis patients (Vonk et al. 2008; Patiwetwitoon et al. 2012).

The purpose of our study was to test whether esophageal dilation, using our previously described CT measurements, is greater in lung diseases thought to be related to aspiration. To do this, we examined the prevalence of esophageal dilation in patients with scleroderma, with and without lung disease, using our previously reported measurements. To provide further evidence for the association of esophageal dilation and lung disease, we included two additional sets of controls, patients from the general pulmonary clinical patients and our previously reported normal subjects.

\section{Materials and Methods}

The study was approved by the Institutional Review Board of the University of Illinois at Chicago. It was performed similarly to a study that was previously reported (Schraufnagel et al. 2008) and the subjects of that study served as normal controls in this study.

\section{Subject groups}

Group 1 was comprised of systemic sclerosis patients with associated interstitial lung disease. This group consists of consecutive systemic sclerosis patients from April 1996 to November 2005 who were referred to the pulmonary clinic in our institution because of significant pulmonary involvement. The diagnosis was confirmed by pulmonologists based on the overall clinical assessment and highresolution CT findings. The diagnosis of systemic sclerosis was made according to the criteria established by American Rheumatism Association (American Rheumatism Association 1980).

Group 2 consisted of systemic sclerosis patients without lung disease. This group consisted of consecutive patients with systemic sclerosis who were seen by a rheumatologist at our institution from April 1996 to November 2005, but were not referred to the pulmonary clinic because they were judged by the rheumatologist to have no clinically significant lung disease. The diagnosis of scleroderma was made according to the criteria established by American Rheumatism Association (American Rheumatism Association 1980). The CT scans of these patients were reviewed and did not show significant changes of interstitial lung disease.

Group 3 consisted of pulmonary clinic patients. This group was randomly selected from consecutive patients seen at the same time, matched by gender and age with group 1 patients in a two to one manner. Patients with collagen vascular disease or esophageal disease were then excluded. Further exclusions were made for individuals who did not have a chest CT scan done at our institution.

Group 4 consisted of normal volunteers who underwent a CT scan to check for calcifications in their coronary arteries. This group was the same population we used to define the normal esophageal characteristics in our previous study (Schraufnagel et al. 2008).

\section{Esophageal measurements on CT scan}

The radiological measurements were performed, as reported in our prior study (Schraufnagel et al. 2008). In brief, images were obtained using a C-100 scanner (GE Imatron, San Francisco, CA, USA) with section thickness of $6 \mathrm{~mm}$. The CT images were set to lung windows (window width 1465 Hounsfield Units (HU), window level $-498 \mathrm{HU}$ ) and stepped downward until one full section showed

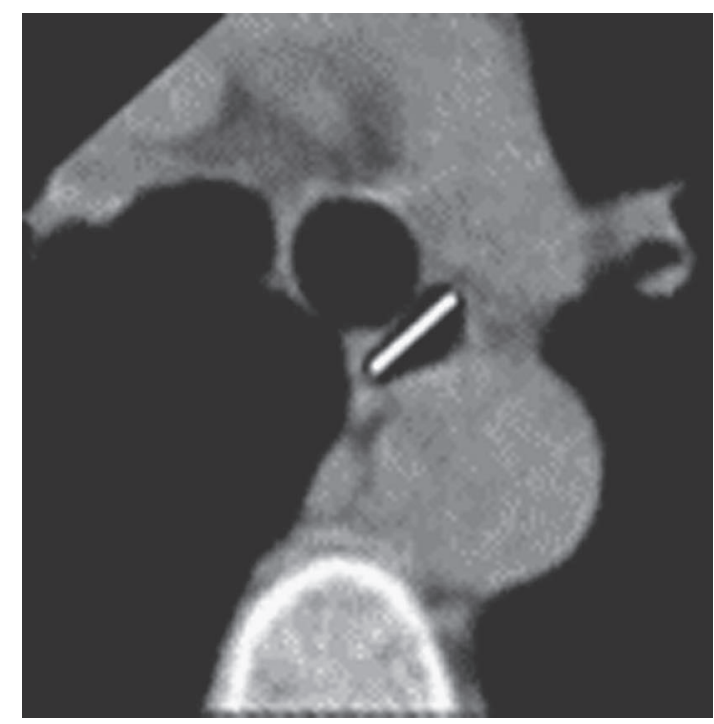

Fig. 1. Measurement of the esophageal diameter. A transectional thoracic CT image is shown, with a white bar indicating how esophageal "diameter" was measured in this study. The longest distance of the esophageal air was measured without touching the wall.

the lung free of the extrapulmonary apical soft tissue. The window setting was then changed to the mediastinal window (window width $396 \mathrm{HU}$, window level $44 \mathrm{HU}$ ). The largest linear measurement of the esophageal air (Dmax) was recorded as the "diameter" (Fig. 1). If the esophagus was folded and had two air densities, only the longer measurement was recorded. The measurement line did not touch the esophageal wall. The largest diameter of esophageal lumen at a constant point, the carina, was recorded for each subject (Dcar). The lower esophageal sphincter (LES) was recorded as closed, if there was no luminal air on the last slice before entrance into the stomach. A hiatal hernia was recorded as being present according to standard criteria: the stomach was identified as being present above the diaphragm by gastric rugae, larger lumen, more bulbous shape, and convergence of the gastric folds above and below the diaphragm (Plavsic et al. 1992). Food was scored as present if heterogeneous material was found at any point in the esophagus. A fluid level was recorded if there was an air-fluid level at any place in the esophagus. The number of sections with the esophagus open (containing air inside) more than $10 \mathrm{~mm}$ was divided by the total number of esophageal sections to give a percentage of the open or dilated esophagus over the whole esophagus (\%Eop).

\section{Scoring of interstitial lung disease}

The CT lung images were evaluated by 2 observers masked to the groupings. For each patient, lungs were divided into the 6 zones: the right and left upper, mid-, and lower lung zones. The upper lung zone was defined as the section at the top of the aortic arch. The midlung zone was defined as the section at the level of the inferior pulmonary veins, and the lower lung zone was defined as the section one cut above the uppermost border of the diaphragm. Each lung zone was scored from 0 to 3 based on a Likert scale in each of the following categories: ground glass, fibrosis (reticular or scarring), honeycombing, and consolidation. Scores signified the following: 0 for no disease evident, 1 for trace or mild disease, 2 for 25 to $50 \%$ of the section involved, 3 for more than $50 \%$ of the section involved. 
The final score for each pathology in a patient was calculated by combining the 6 scores for each lung zone, which could give up to 18 points at maximum.

\section{Pulmonary function tests}

Spirometry, lung volumes, and single-breath diffusion capacity for carbon monoxide were obtained in a standard manner in our pulmonary function laboratory. Only results within three months from the CT scan were used. If there were more than two studies, the study obtained closest to the CT scan was chosen. The results were expressed in percent predicted. The normal group did not have this information.

\section{Data analysis}

Data were expressed by a median with $25-75 \%$ quartile range $(\mathrm{QR})$ unless otherwise indicated. Mann-Whitney $U$ test was used for comparison of the medians. Chi-square or Fisher's exact test was used to compare ratios. Pearson's correlation coefficient was used to assess correlations between pulmonary function tests and esophageal parameters. A p value $<0.05$ was considered significant. All tests were performed on PASW Statistics software, version 18 (IBM, Endicott, NY, USA). The demographic and pulmonary function testing data for the normal group were not available. Statistical comparisons of this information were carried out only in the other 3 groups. We compared the esophageal measurements on CT scans of the groups and correlated the pulmonary function results and esophageal measurements.

\section{Results}

There were 121 systemic sclerosis patients with interstitial lung disease, 48 systemic sclerosis patients without interstitial lung disease, 121 patients from the pulmonary clinic, and 110 normal subjects entered into the study.

The basic patient characteristics are shown in Table 1 . There was no significant difference in age, gender, race, or height in the two systemic sclerosis groups. The age of pulmonary clinic group was older than systemic sclerosis groups $(p<0.01)$. There were fewer male patients in the pulmonary clinic group, but this was not statistically different from the systemic sclerosis groups. This occurred because of exclusions from pulmonary clinic group after initial age- and gender-matched selection.

Comparing systemic sclerosis groups without and with interstitial lung disease

The esophageal comparisons are shown in Table 2 and Fig. 2. Systemic sclerosis patients with interstitial lung disease had larger maximum esophageal diameter than those without lung disease (Dmax $19.4 \mathrm{~mm}$ vs. $14.1 \mathrm{~mm}, \mathrm{p}<$ 0.001 ), diameter at the carina (Dcar $11.2 \mathrm{~mm}$ vs. $5.5 \mathrm{~mm}$, p $=0.005)$, greater overall open esophagi ( $\%$ Eop $51.0 \%$ vs. $22.0 \%, \mathrm{p}<0.001)$, more frequently retained fluid $(28.9 \%$ vs. $8.3, \mathrm{p}=0.004)$ and food $(31.7 \%$ vs. $12.5 \%, \mathrm{p}=0.011)$, and more often open LES ( $28.0 \%$ vs. $4.4, p=0.001)$.

Comparing systemic sclerosis patients with normal subjects

Compared with normal group, the esophagi of systemic sclerosis patients with lung disease and without lung disease were significantly more dilated in all parameters ( $\mathrm{p}$ $<0.01$ ), except for the openness of lower esophageal sphincter in scleroderma patients without lung disease (Table 2, Fig. 2).

Comparing pulmonary clinic patients with normal subjects

Compared with normal subjects, the pulmonary clinic patients had larger maximal diameter (Dmax $12.2 \mathrm{~mm}$ vs. $9.7 \mathrm{~mm}, \mathrm{p}=0.004$ ), diameter at the carina (Dcar $3.1 \mathrm{~mm}$ vs. $0 \mathrm{~mm}, \mathrm{p}=0.002)$, and greater overall open esophagi (\%Eop $9.0 \%$ vs. $0, p=0.001$ ) (Table 2, Fig. 2).

As shown in Table 3, esophageal dilation greater than or equal to $15 \mathrm{~mm}$ (Dmax) occurred in $73.6 \%$ of the systemic sclerosis patients with lung disease, $47.9 \%$ of the systemic sclerosis patients without lung disease, $32.2 \%$ of the pulmonary clinic patients, and $18.2 \%$ of the normal subjects. Diameter at the carina (Dcar) of $10 \mathrm{~mm}$ or greater

Table 1. Patient characteristics.

\begin{tabular}{|c|c|c|c|c|}
\hline Group & $\mathrm{SSc} w / \mathrm{ILD}$ & SSc w/o ILD & Pulm & Normal \\
\hline $\mathrm{N}$ & 121 & 48 & 121 & 110 \\
\hline Age (years) & $\begin{array}{c}51.6 \\
(40.5-60.4)\end{array}$ & $\begin{array}{c}46.2 \\
(38.9-56.1)\end{array}$ & $\begin{array}{c}56.8^{*} \\
(45.2-68.2)\end{array}$ & $\begin{array}{c}51.0 \pm 9.0 \\
(\text { mean } \pm \text { SD })\end{array}$ \\
\hline Male & $26(21.5 \%)$ & $7(14.6 \%)$ & $16(13.2 \%)$ & $63(57.3 \%)$ \\
\hline Race & $\begin{array}{c}\text { B } 37(30.6 \%) \\
\text { H } 16(13.2 \%) \\
\text { W } 56(46.3 \%) \\
\text { O } 11(9.1 \%) \\
\text { unknown } 1(0.8 \%)\end{array}$ & $\begin{array}{c}\text { B } 8(16.7 \%) \\
\text { H } 9(18.8 \%) \\
\text { W } 29(60.4 \%) \\
\text { O } 2(4.2 \%)\end{array}$ & $\begin{array}{c}\text { B } 78(64.5 \%)^{*} \\
\text { H } 23(19 \%) \\
\text { W } 18(14.9 \%) \\
\text { O } 2(1.7 \%)\end{array}$ & - \\
\hline Height (cm) & $163(157-170)$ & $163(156-169)$ & $160(155-168)$ & - \\
\hline
\end{tabular}

SSc w/ ILD: systemic sclerosis patients with interstitial lung disease; SSc w/o ILD: systemic sclerosis patients without interstitial lung disease; Pulm: patients from a pulmonary clinic; Normal: normal subjects. The median and (25\% quartile-75\% quartile) are shown unless otherwise indicated.

$* \mathrm{P}<0.05$ vs. SSc w/ ILD or SSc w/o ILD.

"-_" are not available.

B, Black; H, Hispanic; W, White; O, Others; SD, standard deviation. 
Table 2. Esophageal parameters in four groups.

\begin{tabular}{lcccc}
\hline \multicolumn{1}{c}{ Group } & SSc w/ ILD & SSc w/o ILD & Pulm & Normal \\
\hline Maximal dilation $(\mathrm{mm})$ & $19.4(14.8-25.2)$ & $14.1(10.7-19.4)$ & $12.2(8.4-16.4)$ & $9.7(6.9-14.1)$ \\
& $(\mathrm{N}=121)$ & $(\mathrm{N}=48)$ & $(\mathrm{N}=121)$ & $(\mathrm{N}=110)$ \\
Carina $(\mathrm{mm})$ & $11.2(4.0-16.4)$ & $5.5(1.8-10.9)$ & $3.1(0.0-7.3)$ & $0.0(0.0-4.6)$ \\
& $(\mathrm{N}=120)$ & $(\mathrm{N}=48)$ & $(\mathrm{N}=120)$ & $(\mathrm{N}=110)$ \\
\%Eop (\%) & $51.0(18.0-84.5)$ & $22.0(4.5-43.3)$ & $9.0(0.0-22.0)$ & $0.0(0.0-9.4)$ \\
Esophageal Fluid (\%) & $(\mathrm{N}=121)$ & $(\mathrm{N}=48)$ & $(\mathrm{N}=121)$ & $(\mathrm{N}=110)$ \\
Esophageal Food $(\%)$ & $28.9(\mathrm{~N}=121)$ & $8.3(\mathrm{~N}=48)$ & $1.7(\mathrm{~N}=121)$ & $0.9(\mathrm{~N}=110)$ \\
LES open (\%) & $31.7(\mathrm{~N}=120)$ & $12.5(\mathrm{~N}=48)$ & $2.5(\mathrm{~N}=121)$ & $0.9(\mathrm{~N}=110)$ \\
Hiatal hernia (\%) & $28.0(\mathrm{~N}=118)$ & $4.4(\mathrm{~N}=45)$ & $5.0(\mathrm{~N}=121)$ & $2.8(\mathrm{~N}=109)$ \\
\hline
\end{tabular}

Esophageal parameters in the 4 groups are shown. See Fig. 3 for the p-values. Data are shown in median $(25 \%$ quartile- $75 \%$ quartile) unless otherwise indicated.

SSc w/ ILD: systemic sclerosis patients with interstitial lung disease; SSc w/o ILD: systemic sclerosis patients without interstitial lung disease; Pulm: patients from a pulmonary clinic; Normal: normal subjects.

LES, lower esophageal sphincter; \%Eop, percentage of the length of esophagus whose diameter > 10 mm over full length of esophagus.
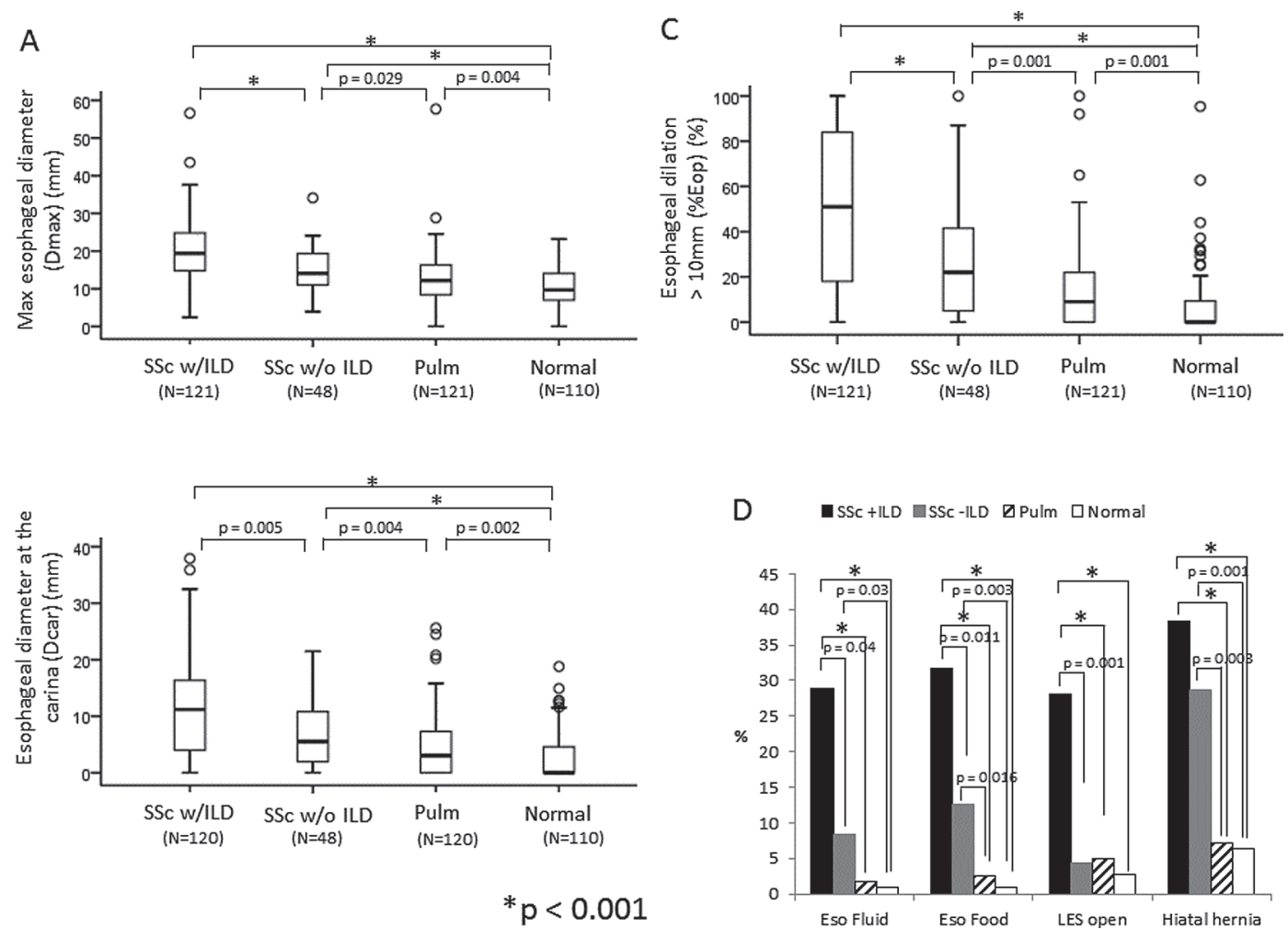

Fig. 2. Esophageal measurements.

The box and whisker plots of 3 measures of the esophageal air column show the median, upper and lower quartiles, and $p$ values of comparisons for each of the 4 groups (A, B, and C). Panel D shows a bar graph of 4 other variables in the 4 groups. "Eso fluid" indicates a fluid level was present in the esophagus. "Eso food" indicates food was found in the esophagus. "LES open" indicates the lower esophageal sphincter was open. Hiatal hernia indicates its presence. Please refer to the Table 2 for the number of subjects in each group for this figure.

was present in $54.2 \%$ of the scleroderma patients with lung disease, $29.2 \%$ of the scleroderma patients without lung disease, and $14.2 \%$ of the patients from the pulmonary clinic, and $10 \%$ of the normal subjects.

\section{Pulmonary function tests}

The results of the pulmonary function tests are shown in Fig. 3; 183 of the $290(63 \%)$ subjects were tested within 3 months of the CT scan. The systemic sclerosis patients with lung disease had worse lung function than the systemic 
Table 3. Prevalence of esophageal dilation.

\begin{tabular}{lccrr}
\hline & SSc w/ ILD & SSc w/o ILD & Pulm & Normal \\
\hline Dmax $\geq 15 \mathrm{~mm}$ & $73.6 \%(89 / 121)$ & $47.9 \%(23 / 48)$ & $32.2 \%(39 / 121)$ & $18.2 \%(20 / 110)$ \\
Dcar $\geq 10 \mathrm{~mm}$ & $54.2 \%(65 / 120)$ & $29.2 \%(14 / 48)$ & $14.2 \%(17 / 120)$ & $10 \%(11 / 110)$ \\
\hline
\end{tabular}

SSc w/ ILD: systemic sclerosis patients with interstitial lung disease; SSc w/o ILD: systemic sclerosis patients without interstitial lung disease; Pulm: patients from a pulmonary clinic; Normal: normal subjects; Dmax: maximal esophageal air column width; Dcar: esophageal air column width at the carina level.
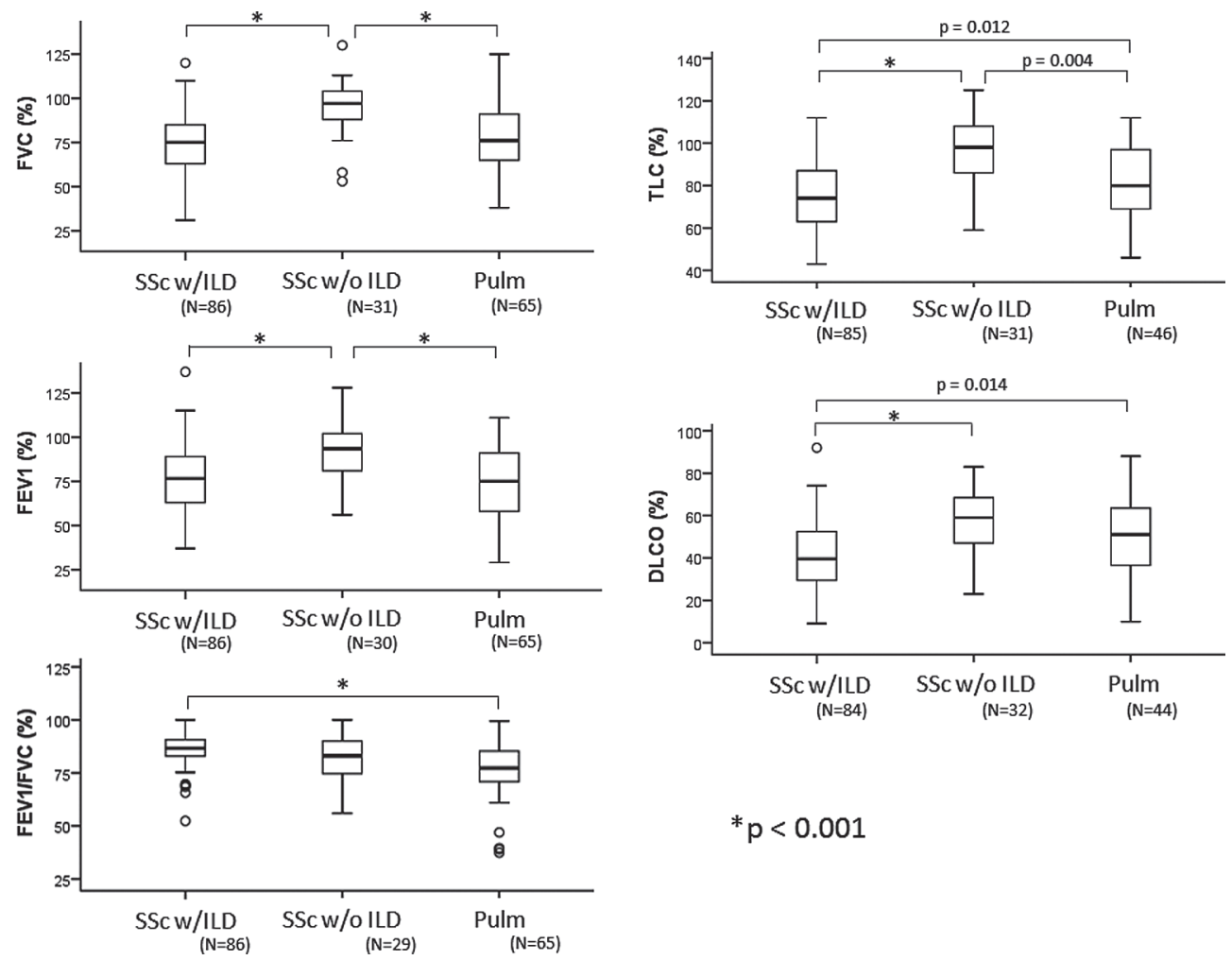

$$
{ }^{*} p<0.001
$$

Fig. 3. Pulmonary function tests.

The box and whisker plots of 5 pulmonary function tests show the median, upper and lower quartiles, and $p$ values of comparisons for each of the 3 groups that had pulmonary function tests recorded. The total 183 subjects were tested within 3 months of the CT scan: 86 systemic sclerosis patients with interstitial lung disease (SSc w/ ILD), 32 systemic sclerosis patients without interstitial lung disease (SSc w/o ILD), and 65 patients from the pulmonary clinic (Pulm). The numbers of the patients in each PFT parameter are different, because the data were missing for several patients for some parameters in the medical record.

sclerosis patients without lung disease in all parameters, except in forced expiratory volume in 1 second divided by forced vital capacity (FEV1/FVC). The systemic sclerosis patients without interstitial lung disease also had better FVC, FEV1, and total lung capacity (TLC), compared with pulmonary clinic group. The systemic sclerosis patients with interstitial lung disease had higher FEV1/FVC, lower TLC, and lower diffusing capacities (DLCO) compared with pulmonary group.

Correlation between pulmonary function tests and esophageal measurements in systemic sclerosis and pulmonary clinic groups

The correlation coefficients for esophageal measurements and pulmonary function tests are shown in Table 4.

In systemic sclerosis patients, the maximal esophageal diameter (Dmax) negatively correlated with FVC, TLC, and DLCO. The diameter at the carina (Dcar) negatively correlated with DLCO. The percent dilated (\%Eop) negatively correlated with FVC, FEV1, TLC, and DLCO. There was a positive correlation between maximal diameter (Dmax) and FEV1/FVC.

In pulmonary clinic patients, maximal diameter (Dmax) negatively correlated with FVC and positively correlated with FEV1/FVC. Diameter at the carina (Dcar) correlated with FEV1/FVC and DLCO. 
Table 4. Correlation between esophageal measurements and pulmonary function tests in patients with systemic sclerosis.

\begin{tabular}{|c|c|c|c|c|c|c|c|c|c|}
\hline \multicolumn{10}{|c|}{ Systemic sclerosis patients } \\
\hline & \multicolumn{3}{|c|}{ Dmax } & \multicolumn{3}{|c|}{ Dcar } & \multicolumn{3}{|c|}{$\%$ Eop } \\
\hline & $\mathrm{r}$ & $\mathrm{p}$ & $\mathrm{N}$ & $\mathrm{r}$ & $\mathrm{p}$ & $\mathrm{N}$ & $\mathrm{r}$ & $\mathrm{p}$ & $\mathrm{N}$ \\
\hline FVC & -0.187 & 0.016 & 164 & -0.049 & 0.531 & 163 & -0.224 & 0.004 & 164 \\
\hline FEV1 & -0.106 & 0.183 & 160 & 0.008 & 0.919 & 159 & -0.175 & 0.027 & 160 \\
\hline FEV1/FVC & 0.166 & 0.037 & 158 & 0.080 & 0.319 & 157 & 0.120 & 0.132 & 158 \\
\hline TLC & -0.201 & 0.010 & 161 & -0.090 & 0.259 & 160 & -0.209 & 0.008 & 161 \\
\hline DLCO & -0.263 & 0.001 & 162 & -0.239 & 0.002 & 161 & -0.314 & $<0.001$ & 162 \\
\hline \multicolumn{10}{|c|}{ Pulmonary clinic patients } \\
\hline & \multicolumn{3}{|c|}{ Dmax } & \multicolumn{3}{|c|}{ Dcar } & \multicolumn{3}{|c|}{$\%$ Eop } \\
\hline & $\mathrm{r}$ & $\mathrm{p}$ & $\mathrm{N}$ & r & $\mathrm{p}$ & $\mathrm{N}$ & r & $\mathrm{p}$ & $\mathrm{N}$ \\
\hline FVC & -0.199 & 0.032 & 116 & -0.052 & 0.579 & 115 & -0.130 & 0.166 & 116 \\
\hline FEV1 & -0.039 & 0.676 & 116 & 0.077 & 0.413 & 115 & -0.013 & 0.894 & 116 \\
\hline FEV1/FVC & 0.203 & 0.029 & 116 & 0.187 & 0.046 & 115 & 0.139 & 0.136 & 116 \\
\hline TLC & -0.04 & 0.702 & 92 & 0.100 & 0.346 & 91 & -0.041 & 0.7 & 92 \\
\hline DLCO & 0.121 & 0.260 & 88 & 0.289 & 0.007 & 87 & 0.088 & 0.417 & 88 \\
\hline
\end{tabular}

Dmax: maximal esophageal luminal diameter; Dcar: esophageal luminal diameter at the level of carina.

\%Eop, percentage of esophagus whose luminal diameter > $10 \mathrm{~mm}$ over the full length of the esophagus; FVC, forced vital capacity;

FEV1, forced expiratory volume at 1 second; TLC, total lung capacity; DLCO, diffusion capacity for carbon monoxide.

Table 5. Scores of the interstitial lung disease in the three groups.

\begin{tabular}{lcccccc}
\hline & SSc w/ ILD & SSc w/o ILD & Pulm & $\begin{array}{c}\text { SSc w/ ILD } \\
\text { vs. SSc w/o ILD }\end{array}$ & $\begin{array}{c}\text { SSc w/ ILD } \\
\text { vs. Pulm }\end{array}$ & $\begin{array}{c}\text { SSc w/o ILD } \\
\text { vs. Pulm }\end{array}$ \\
\hline Ground glass & $4(1-6)$ & $1(0-2)$ & $2(0-6)$ & $\mathrm{p}<0.001$ & $\mathrm{p}=0.084$ & $\mathrm{p}=0.001$ \\
Reticulation or fibrosis & $3(0-7)$ & $0(0-1.75)$ & $1(0-4)$ & $\mathrm{p}<0.001$ & $\mathrm{p}=0.016$ & $\mathrm{p}=0.001$ \\
Honeycombing & $0(0-1)$ & $0(0-0)$ & $0(0-0)$ & $\mathrm{p}=0.001$ & $\mathrm{p}<0.001$ & $\mathrm{p}=0.429$ \\
Consolidation & $0(0-0)$ & $0(0-0)$ & $0(0-0)$ & $\mathrm{p}=0.415$ & $\mathrm{p}=0.010$ & $\mathrm{p}=0.005$ \\
\hline
\end{tabular}

SSc w/ ILD: systemic sclerosis patients with interstitial lung disease; SSc w/o ILD: systemic sclerosis patients without interstitial lung disease; Pulm: patients from a pulmonary clinic.

The lungs were evaluated in the following six lung zones: the right and left upper, mid-, and lower lung zones. Each zone was scored from 0-3 on a Likert scale for ground glass, fibrosis, honeycombing, and consolidation. A combined lung score was calculated for each category of pathology and reported. The lung scores ( 0 to 3$)$ for all the 6 zones could give up to 18 points at maximum. The median and ( $25 \%$ quartile- $75 \%$ quartile) are shown unless otherwise indicated.

\section{Interstitial lung disease}

The interstitial lung disease scoring showed significantly greater ground glass, reticular, and honeycombing opacities in the patients in the systemic sclerosis with lung disease group than the other groups (Table 5). Consolidation was rare in all patients and honeycombing was rare in all except the systemic sclerosis patients with lung disease group.

\section{Discussion}

In this paper we tested our CT esophageal measurements on a group of patients with known esophageal disease (systemic sclerosis) and sought differences between those with and without lung disease against the standard normal subjects and a group of matched patients from the general pulmonary clinic. We found that systemic sclerosis patients with lung disease had more dilated esophagi than systemic sclerosis patients without lung disease. Both of these groups had more dilated esophagi than pulmonary clinic patients, but the pulmonary clinic patients had more esophageal dilatation than normal.

It is well known that the esophagus is dilated in systemic sclerosis (Bhalla et al. 1993; Pitrez et al. 2006; Vonk et al. 2008; Patiwetwitoon et al. 2012), but its precise measurements and association with lung disease had not been done before. The finding of retained food and liquid also indicates esophageal dysfunction. CT studies have called esophageal dilation with coronal lumen more than $10 \mathrm{~mm}$ (Bhalla et al. 1993; Vonk et al. 2008) or the widest coronal esophageal diameter at 3 levels (the mid-arch of the aorta, the main carina, and the diaphragmatic hiatus) more than 10 mm (Patiwetwitoon et al. 2012). Our previous report 
recorded the esophageal diameter in a normal population and found the esophagus is likely to be dilated in its lower third (Schraufnagel et al. 2008). We proposed a cutoff value of $10 \mathrm{~mm}$ for the carinal level and $15 \mathrm{~mm}$ for maximum diameter. The prevalence defined by maximal diameter (Dmax) was consistently higher than carinal diameter (Dcar), indicating maximal diameter is more sensitive but less specific criteria for esophageal dilation.

Accumulating evidence suggests an important role of esophageal dysfunction in the development of lung disease in systemic sclerosis or other lung diseases (el-Serag and Sonnenberg 1997; Tobin et al. 1998; Marie et al. 2001; Savarino et al. 2009; Christmann et al. 2010; Patiwetwitoon et al. 2012). In this study, we found a correlation between esophageal parameters and pulmonary function tests in systemic sclerosis patients. Systemic sclerosis patients with lung disease had more dilated esophagi and worse pulmonary function tests than systemic sclerosis patients without lung disease.

Surprisingly, general pulmonary clinic patients had more dilated esophagus than normal, findings that support the potential role of esophageal dysfunction in the development of other lung diseases. The most common diseases in the general pulmonary clinic were chronic obstructive pulmonary disease, asthma, lung cancer, and symptom-related complaints such as chest pain, dyspnea, and cough. The chest CTs in the pulmonary clinic patients were often abnormal, but interstitial lung disease was uncommon as shown in Table 5.

Not surprisingly, the interstitial lung disease scoring was several times higher in the systemic sclerosis patients with lung disease than those without. It also showed a mix of disease in the pulmonary clinic patients. The lower FEV1/FVC in the pulmonary clinic patients confirmed their higher prevalence of obstructive disease.

Esophageal dysfunction in patients with systemic sclerosis or other lung diseases is seldom detected at early stage (Ing 2001; Raghu 2003; Gaude 2009; Christmann et al. 2010; Soares et al. 2011), probably because reflux disease is often clinically silent (Raghu 2003) and diagnostic tests such as manometry, $\mathrm{pH}$ and impedance monitoring, endoscopy, and scintigraphy, are invasive or not routinely performed in everyday practice. Using CT scan measurements has several advantages in assessing esophageal dysfunction over the conventional methods. It is non-invasive, widely used, and easily repeatable. In addition, CT imaging is often already available, because chest CT scans are commonly performed as part of clinical evaluation of the patients with systemic sclerosis. Therefore, by detecting patients with esophageal dysfunction with CT scans at early stage of interstitial lung disease, it may be possible to initiate early treatment and prevent further progression of the lung disease. The current study was not longitudinal study and, hence, was unable to evaluate this possibility.

There are several limitations in this study. First, as was mentioned above, it was a retrospective cross-sectional study, which could not prove causality of esophageal dysfunction on the lung involvement. Second, it is possible that systemic sclerosis patients with interstitial lung disease had more dilated esophagi merely because these patients had a more severe disease that directly involved both the lung and esophagus. However, we demonstrated pulmonary clinic patients also were more likely to have dilated esophagi, even after excluding patients with known esophageal and rheumatologic diseases. This suggests that a simple severity of systemic sclerosis could not fully explain the difference. Third, even though the pulmonary clinic patients were initially age- and sex-matched with scleroderma group, more than expected exclusions made the matching less even. The pulmonary clinic group was older than scleroderma groups. However, age was not a statistically significant contributor for esophageal dilation in our previous study (Schraufnagel et al. 2008). In addition, there were significantly more male patients in the normal group. Women have slightly smaller esophageal diameters than men do, although the difference was not statistically significant (Schraufnagel et al. 2008). This would affect the comparison between patient groups and normal subjects in the direction of non-significance. However, there actually was a strongly significant difference. Hence, it did not affect the conclusions.

In summary, we demonstrated that patients with systemic sclerosis and interstitial lung disease had more dilated esophagi on CT scan compared with patients with systemic sclerosis and no significant lung disease, which is consistent with the hypothesis that esophageal dysfunction has a role in development of lung disease in this group. The pulmonary clinic patients also had more dilated esophageal air columns on CT compared with normal subjects, supporting the contribution of esophageal dysfunction in the development of lung diseases in general. CT measurements of the esophagus may be useful for the routine clinical workup. Future studies should test the esophageal air column size as a biomarker for esophageal dysfunction and the development of lung disease.

\section{Conflict of Interest}

The authors declare no conflict of interest.

\section{References}

American Rheumatism Association (1980) Preliminary criteria for the classification of systemic sclerosis (scleroderma). Subcommittee for scleroderma criteria of the American Rheumatism Association Diagnostic and Therapeutic Criteria Committee. Arthritis Rheum., 23, 581-590.

Bhalla, M., Silver, R.M., Shepard, J.A. \& McLoud, T.C. (1993) Chest CT in patients with scleroderma: prevalence of asymptomatic esophageal dilatation and mediastinal lymphadenopathy. AJR Am. J. Roentgenol., 161, 269-272.

Christmann, R.B., Wells, A.U., Capelozzi, V.L. \& Silver, R.M. (2010) Gastroesophageal reflux incites interstitial lung disease in systemic sclerosis: clinical, radiologic, histopathologic, and treatment evidence. Semin. Arthritis Rheum., 40, 241-249.

el-Serag, H.B. \& Sonnenberg, A. (1997) Comorbid occurrence of 
laryngeal or pulmonary disease with esophagitis in United States military veterans. Gastroenterology, 113, 755-760.

Gaude, G.S. (2009) Pulmonary manifestations of gastroesophageal reflux disease. Ann. Thorac. Med., 4, 115-123.

Ing, A.J. (2001) Interstitial lung disease and gastroesophageal reflux. Am. J. Med., 111 Suppl 8A, 41S-44S.

Marie, I., Dominique, S., Levesque, H., Ducrotte, P., Denis, P., Hellot, M.F. \& Courtois, H. (2001) Esophageal involvement and pulmonary manifestations in systemic sclerosis. Arthritis Rheum., 45, 346-354.

Patiwetwitoon, S., Wangkaew, S., Euathrongchit, J., Kasitanon, N. \& Louthrenoo, W. (2012) High-resolution computed tomographic findings in systemic sclerosis-associated interstitial lung disease: comparison between diffuse and limited systemic sclerosis. J. Clin. Rheumatol., 18, 229-233.

Pitrez, E.H., Bredemeier, M., Xavier, R.M., Capobianco, K.G., Restelli, V.G., Vieira, M.V., Ludwig, D.H., Brenol, J.C., Furtado, A.P., Fonseca, L.M. \& Gutfilen, B. (2006) Oesophageal dysmotility in systemic sclerosis: comparison of HRCT and scintigraphy. Br. J. Radiol., 79, 719-724.

Plavsic, B.M., Robinson, A.E. \& Jeffrey, R.B. (1992) Gastrointestinal Radiology: A Concise Text, McGraw-Hill, New York, NY.

Raghu, G. (2003) The role of gastroesophageal reflux in idiopathic pulmonary fibrosis. Am. J. Med., 115 Suppl 3A, 60S-64S.

Reveille, J.D. (2003) Ethnicity and race and systemic sclerosis: how it affects susceptibility, severity, antibody genetics, and clinical manifestations. Curr. Rheumatol. Rep., 5, 160-167.

Savarino, E., Bazzica, M., Zentilin, P., Pohl, D., Parodi, A., Cittadini, G., Negrini, S., Indiveri, F., Tutuian, R., Savarino, V. \& Ghio, M. (2009) Gastroesophageal reflux and pulmonary fibrosis in scleroderma: a study using $\mathrm{pH}$-impedance monitoring. Am. J. Respir. Crit. Care Med., 179, 408-413.

Schraufnagel, D.E., Michel, J.C., Sheppard, T.J., Saffold, P.C. \& Kondos, G.T. (2008) CT of the normal esophagus to define the normal air column and its extent and distribution. AJR Am. J. Roentgenol., 191, 748-752.

Soares, R.V., Forsythe, A., Hogarth, K., Sweiss, N.J., Noth, I. \& Patti, M.G. (2011) Interstitial lung disease and gastroesophageal reflux disease: key role of esophageal function tests in the diagnosis and treatment. Arq. Gastroenterol., 48, 91-97.

Steen, V.D. \& Medsger, T.A. (2007) Changes in causes of death in systemic sclerosis, 1972-2002. Ann. Rheum. Dis., 66, 940944.

Tobin, R.W., Pope, C.E. 2nd., Pellegrini, C.A., Emond, M.J., Sillery, J. \& Raghu, G. (1998) Increased prevalence of gastroesophageal reflux in patients with idiopathic pulmonary fibrosis. Am. J. Respir. Crit. Care Med., 158, 1804-1808.

Vonk, M.C., van Die, C.E., Snoeren, M.M., Bhansing, K.J., van Riel, P.L., Fransen, J. \& van den Hoogen, F.H. (2008) Oesophageal dilatation on high-resolution computed tomography scan of the lungs as a sign of scleroderma. Ann. Rheum. Dis., 67, 1317-1321. 